'Departamento de Solos e Engenharia Rural, Centro de Ciências Agrárias, Universidade Federal da Paraíba - UFPB, Rodovia BR 079, Km 12 Campus Universitário, CEP 58397 000, Areia, PB, Brasil

${ }^{2}$ Centro de Ciências e Tecnologia Agroalimentar, Universidade Federal de Campina Grande - UFCG, Pombal, PB, Brasil

${ }^{3}$ Departamento de Agroindūstria, Instituto Federal de Alagoas, Piranhas, AL, Brasi

${ }^{4}$ Universidade Catôlica de Pernambuco - UNICAP, Recife, PE, Brasil

Departamento de Ciências Atmosféricas, Centro de Tecnologia e Recursos Naturais, Universidade Federal de Campina Grande - UFCG Campina Grande, PB, Brasil ${ }^{6}$ Centro de Ciências Agrárias, Universidade Federal da Paraíba UFPB, Areia, PB, Brasil 'Departamento de Química Biológica, Universidade Regional do Cariri URCA, Crato, CE, Brasil 'Departamento de Ciências Atmosféricas, Centro de Tecnologia e Recursos Naturais, Universidade Federal de Campina Grande - UFCG, Campina Grande, PB, Brasil

* autor correspondente saul.oliveira.ramos@hotmail.com

\title{
Produtividade e crescimento de banana 'Nanica' em função de doses de nitrogênio e esterco bovino
}

\author{
Productivity and growth of banana 'nanica' as a function of \\ nitrogen and bovine manure doses
}

Saul Ramos de Oliveira ${ }^{*}$, Josinaldo Lopes Araújo², Flaubert Queiroga de Sousa²,

Romário Oliveira de Andrade ${ }^{3}$, Caio Franklin Vieira de Figueiredo ${ }^{4}$, Francisco Fabrício Damião de Oliveira 5 , Raiff Ramos Almeida Nascimento ${ }^{6}$. Aliane Cristiane de Sousa Formiga ${ }^{7}$, Glaúcio de Meneses Sousa ${ }^{8}$

RESUMO: O trabalho teve como objetivo avaliar o efeito de doses de nitrogênio sobre o crescimento e produção da bananeira (Musa ssp.), com ou sem a aplicação de esterco bovino. $\mathrm{O}$ experimento foi conduzido na Fazenda Riachão, localizada no município de Pombal-PB. O delineamento experimental foi em blocos casualizados em esquema fatorial $4 \times 2$, sendo quatro doses de nitrogênio $(0,60,120$ e $180 \mathrm{~kg} \mathrm{~N} / \mathrm{ha})$ e duas doses de matéria orgânica ( 0 e $15 \mathrm{~L}$ por cova). Cada repetição foi constituída por sete plantas, sendo tomadas para as medições apenas as cinco plantas centrais de cada bloco. As mudas de bananeira (Musa ssp.), variedade 'Nanica' foram plantadas no espaçamento $2 \mathrm{~m} \times 3 \mathrm{~m}$, em covas de $40 \mathrm{~cm}$ x $40 \mathrm{~cm} \mathrm{x} 40 \mathrm{~cm}$ de profundidade. $\mathrm{Na}$ fase de crescimento vegetativo (90 dias após o plantio) e no início do florescimento (270 dias), foram avaliados a altura de plantas, o diâmetro do caule e a produtividade do frutos. Pelos resultados, constatou-se que apenas a produtividade foi afetada pelas doses de nitrogênio. Concluiu-se que o crescimento da bananeira 'nanica' não foi influenciado positivamente pela adubação nitrogenada, no entanto, foi favorecida pela aplicação de esterco bovino. A máxima produtividade da banana 'nanica' foi obtida com a aplicação de $82,3 \mathrm{~kg} \mathrm{~N} / \mathrm{ha}$ sem a aplicação de esterco bovino.

PALAVRAS-CHAVE: Adubação orgânica, esterco bovino, doses de nitrogênio.
ABSTRACT: The work aimed to evaluate the effect of nitrogen doses on the growth and production of banana (Musa ssp.), with or without the application of bovine manure. The experiment was carried out at Fazenda Riachão, located in the municipality of Pombal-PB. The experimental design was randomized blocks in a $4 \times 2$ factorial scheme, with four doses of nitrogen (0, 60, 120 and $180 \mathrm{~kg} \mathrm{~N} / \mathrm{ha}$ ) and two doses of organic matter (0 and $15 \mathrm{~L}$ per pit). Each replication was composed of seven plants, with only five first plants of each block being measured. The seedlings (Musa ssp.) of the variety 'nanica'were planted at $2 \mathrm{~m} \times 3 \mathrm{~m}$ spacing in $40 \mathrm{~cm} \times 40 \mathrm{~cm} \times 40 \mathrm{~cm}$ deep pits. During the vegetative growth phase (90 days after planting) and at the beginning of flowering (270 days), plant height, stem diameter and fruit productivity were obtained. The results showed that only productivity was affected by nitrogen doses. We conclude that the growth of banana 'nanica' was not positively influenced by nitrogen fertilization; however, it was favored by the application of bovine manure. The maximum productivity of the banana 'nanica'was obtained with the application of $82.3 \mathrm{kgN} / \mathrm{ha}$ without the application of bovine manure.

KEYWORDS: Organic fertilization, bovine manure, nitrogen doses.

\section{Introdução}

A banana (Musa spp.) é uma das frutas mais consumidas no mundo na forma fresca, cultivada na maioria dos países tropicais e de Norte a Sul do Brasil, garantindo emprego e renda para milhares de brasileiros (OLIVEIRA; SOUZA, 2003). A cultura da banana assume importância econômica e social em todo o mundo, sendo cultivada em mais de 80 países tropicais, 
principalmente por agricultores de pequenas propriedades (GONÇALVES et al., 2008).

A banana é apreciada por pessoas de todas as classes sociais e de qualquer idade, que a consomem in natura, frita, assada, cozida, em doces caseiros ou em produtos industrializados, como: passas, doces, chips, polpas e álcool. Segundo a Organização das Nações Unidas para a Alimentação e a Agricultura (FAO), em 2017 a banana foi produzida em 128 países, e é uma das frutas mais consumidas no mundo. Em 2017, a produção mundial de banana atingiu aproximadamente 125,3 milhões de toneladas. Os quatro maiores produtores foram: Índia com 30,5 milhões de toneladas, China com 22,8 milhões de toneladas, Indonésia com 7,2 milhões de toneladas, e Brasil com 6,7 milhões de toneladas. O maior consumidor mundial da fruta é a China.

No Brasil, a bananicultura é desenvolvida em ecossistemas variados, com predominância de diferentes fatores de estresse abióticos. O crescimento e o desenvolvimento da bananeira, e sua consequente produtividade de frutos, são processos dependentes das interações água-solo-genótipo-atmosfera e da interferência humana. Em 2017, a área plantada no país atingiu 469,5 mil hectares, a quantidade produzida foi de 6,7 milhões de toneladas e o total do valor da produção atingiu R \$ 8,1 bilhões (INSTITUTO BRASILEIRO DE GEOGRAFIA E ESTATÍSTICA, 2019). No Brasil, o consumo é estimado em $25 \mathrm{~kg}$ per capita ao ano, representando $0,87 \%$ das despesas de alimentação do brasileiro. Cerca de $98 \%$ da produção é destinada ao consumo in natura, sendo o restante da produção representada pela fruta processada.

Como forma de aumentar a expressão produtiva e atender às exigências nutricionais da cultura, a prática da adubação requer o fornecimento de nutrientes na forma adequada e na quantidade desejada pela cultura (BRAZIL et al., 2000). Dentre os princípios básicos do cultivo orgânico, inclui-se a proteção do solo, do homem e do meio ambiente, favorecendo o desenvolvimento rural e contribuindo para melhores condições de vida (SARUDI et al., 2003). A exploração orgânica de fruteiras tropicais com ênfase para a bananeira é de fundamental importância na programação de uma alimentação equilibrada e, consequentemente, para a saúde humana (GUERRA et al., 2007). Borges et al. (2002) observaram efeitos positivos da adubação orgânica em bananeiras em que o uso de esterco como fonte de adubo promoveu aumento do número e comprimento de frutos por cacho.

O nitrogênio $(\mathrm{N})$ exerce influência em todas as fases da cultura, sendo fundamental para o crescimento vegetativo da planta, sobretudo nos três primeiros meses, quando o meristema está em desenvolvimento e também na fase de produção (BORGES; OLIVEIRA, 2000). O N é responsável pelo aumento do número de folhas e suas dimensões, pelo número de pencas e de frutos de banana, pela emissão e crescimento dos rebentos. Contudo, aplicação de doses adequadas é de grande importância, pois o excesso de $\mathrm{N}$ atrasa a emergência do cacho, o que favorece a produção de cachos fracos e pencas espaçadas (BORGES; OLIVEIRA, 2000; SILVA et al., 2003). A hipótese deste estudo é que a aplicação de nitrogênio, associada à adubação orgânica com uso de esterco bovino, exerce influência positiva no aumento e na produção de frutos da bananeira. Em vista dos altos preços dos fertilizantes químicos e da baixa capitalização de pequenos bananicultores, o uso de fertilizantes orgânicos, muitas vezes encontrados em suas propriedades, torna-se uma ótima alternativa na redução de custos com insumos. Dessa forma, o objetivo com este trabalho foi avaliar o efeito de doses de nitrogênio sobre o crescimento e produção da bananeira com ou sem a aplicação de esterco bovino.

\section{Material e Métodos}

O experimento foi conduzido na Fazenda Riachão, localizada nomunicípio de Pombal-PB. A área está situada nas coordenadas geográficas $06^{\circ} 52^{\prime} 16,1^{\prime \prime}$ Latitude e $37^{\circ} 49^{\prime} 45,3^{\prime \prime}$ W Longitude, distando $13 \mathrm{~km}$ da cidade de Pombal, no estado da Paraíba. Segundo a classificação de Köppen, o clima é caracterizado com AW', temperatura média anual de $28^{\circ} \mathrm{C}$.

$\mathrm{O}$ delineamento experimental foi em blocos casualizados em esquema fatorial $4 \times 2$, sendo quatro doses de nitrogênio $(0,60,120$ e $180 \mathrm{~kg} \mathrm{~N} / \mathrm{ha})$ e duas doses de matéria orgânica (0 e $15 \mathrm{~L}$ por cova), totalizando oito tratamentos com três repetições. Cada repetição foi constituída por sete plantas, sendo tomadas para as medições apenas as cinco plantas centrais de cada bloco. Empregou-se como fonte de nitrogênio o sulfato de amônio, e como fonte de matéria orgânica o esterco bovino com as concentrações totais de $\mathrm{N}, \mathrm{P}_{2} \mathrm{O}_{5}$ e $\mathrm{K}_{2} \mathrm{O}$, de 1,7\%, $0,9 \%$ e $1,4 \%$, respectivamente.

O solo é classificado como Nesossolo Flúvico (EMBRAPA, 2006), cujos atributos químicos obtidos na camada de 0-40 cm. As mudas de bananeira (Musa ssp.), variedade 'Nanica' na fase "chifrinho", foram selecionadas conforme a altura e plantadas no espaçamento $2 \mathrm{~m} \mathrm{x} 3 \mathrm{~m}$, em covas de $40 \mathrm{~cm}$ x $40 \mathrm{~cm}$ x 40 $\mathrm{cm}$. Antes do plantio, todas as covas receberam uma adubação com $60 \mathrm{~g}$ de $\mathrm{P}_{2} \mathrm{O}_{5}$ e $60 \mathrm{~g} \mathrm{~K}_{2} \mathrm{O}$, embora os teores de fósforo e potássio tenham se mostrado elevados, como demonstrou a análise de solo (Tabela 1).

$\mathrm{Na}$ fase de crescimento vegetativo (90 dias após o plantio) e no início do florescimento (270 dias após o plantio), foram realizadas medições do diâmetro do pseudocaule a $20 \mathrm{~cm}$ do solo e altura de planta. Na época de produção, foram retirados e pesados os cachos de frutos das cinco plantas centrais para obtenção da produção em $\mathrm{kg} / \mathrm{ha}$. As variáveis foram avaliadas

Tabela 1. Atributos químicos para fins de fertilidade do solo onde foi realizado o experimento.

\begin{tabular}{|c|c|c|c|c|c|c|c|c|c|c|c|}
\hline \multirow{2}{*}{$\mathrm{pH}\left(\mathrm{H}_{2} \mathrm{O}\right)$} & $\mathbf{P}$ & $\mathbf{K}$ & $\mathrm{Na}$ & \multirow{2}{*}{$\mathrm{Ca}$} & Mg & Al & \multirow{2}{*}{$\mathbf{H}+\mathbf{A l}$} & \multirow{2}{*}{ SB } & \multirow{2}{*}{ CTC } & $\mathbf{V}$ & \multirow{2}{*}{$\frac{\text { M.O }}{\text { gkg }^{-1}}$} \\
\hline & \multicolumn{3}{|c|}{$\mathrm{mg} \mathrm{dm^{-3 }}$} & & \multicolumn{2}{|c|}{$\mathrm{cmol}_{\mathrm{c}} \mathrm{dm}^{-3}$} & & & & $\%$ & \\
\hline 6,5 & 124 & 0,31 & $\mathbf{0 , 0 7}$ & 4,5 & 1,9 & $\mathbf{0 , 0}$ & 0,66 & 6,8 & 7,5 & 91 & 3,7 \\
\hline
\end{tabular}

P, K, Na Extrator Mehlich 1; Al, Ca, Mg; Extrator $\mathrm{KCl} 1 \mathrm{M} ; \mathrm{SB}=\mathrm{Ca}^{+2}+\mathrm{Mg}^{2+}+\mathrm{Na}^{+}+\mathrm{K}^{+}: \mathrm{H}+\mathrm{Al}$ : Extrator Acetato de Cálcio 0,5 M, pH 7,0 CTC=SB+H +Al+3: $\mathrm{M} . \mathrm{O}$ :

Digestão via úmida Walkley-Black. V: $\mathrm{V} \%=[$ Soma de bases $(\mathrm{K}+\mathrm{Ca}+\mathrm{Mg}+\mathrm{Na}) \times 100] / \mathrm{CTC}$. 
mediante análise de regressão polinomial para as doses de nitrogênio e teste de médias Tukey para doses de matéria orgânica ao nível de 5\% de significância pelo SAS (2001).

\section{Resultados e Discussão}

As variáveis altura de planta e diâmetro do pseudocaule, em ambas as épocas de avaliação, não foram influenciadas pelas doses de nitrogênio (N) aplicadas (Figura 1). Contudo, a aplicação de esterco bovino na cova favoreceu estas variáveis em todas as doses de $\mathrm{N}$ aplicadas. Embora estas variáveis não tenham sido alteradas pelas doses de $\mathrm{N}$ aplicadas, os valores de altura estiveram acima dos esperados para a variedade "nanica, enquanto o diâmetro se mostrou normal para a variedade (BORGES; OLIVEIRA, 2000; SILVA; FLORES; LIMA NETO, 2002). Assim, o nitrogênio não foi limitante ao crescimento das plantas, mas a adição de esterco proporcionou um desequilíbrio entre estas características, o que foi constatado em campo na fase de produção, pelo tombamento de algumas plantas, que não suportaram o peso do cacho.

Cavalcante et al. (2010) e Oliveira et al. (2010) também constataram que o desequilíbrio nutricional provocado pelo excesso de nitrogênio afeta o crescimento da bananeira. Esses autores verificaram que, após dose ótima de biofertilizante, que seria em torno de $0,80 \mathrm{~L} /$ planta/vez, ocorre redução do crescimento do pseudocaule a partir dessa dosagem.

\section{0 dias após o trasnplantio}

(A)

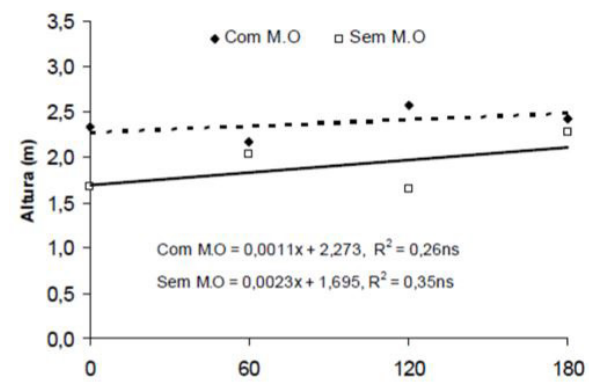

(C)

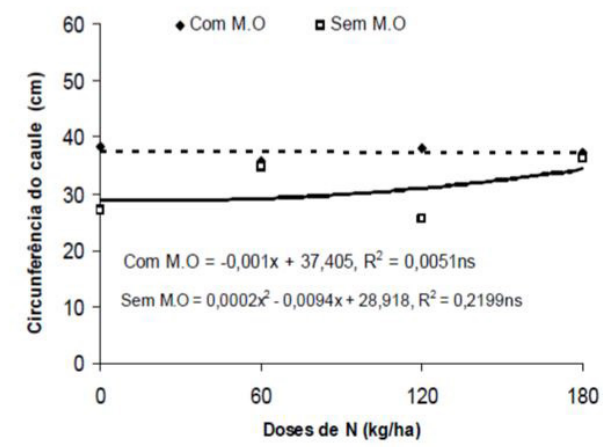

Borges et al. (2002) obtiveram resultados interessantes, em que, no primeiro ano de cultivo (média de 528 dias), a adubação nitrogenada influenciou na altura da planta, número de frutos por cacho e o comprimento e diâmetro médio do fruto; no entanto, doses crescentes de nitrogênio não tiveram efeitos sobre a produção da cultura, mas a adubação orgânica proporcionou um aumento de frutos por cacho e o comprimento médio do fruto.

A produtividade da bananeira 'nanica' foi influenciada pelas doses de $\mathrm{N}$ e pela aplicação de esterco bovino na cova. Esta variável ajustou-se ao modelo de regressão quadrática positiva e negativa, com a ausência e presença de esterco bovino, respectivamente (Figura 2).

Resultados diferentes foram descritos por Guimarães et al. (2020), testando três fontes de fertilizantes (mineral, mineral + composto orgânico ou orgânico-mineral) na cultura da banana. Foi visto nesse estudo que a fertilização mineral proporcionou maior produtividade dos frutos de que as fontes organomineral; no entanto, os autores indicaram que as fontes organominerais acidificaram menos o solo, bem como disponibilizaram maiores teores de $\mathrm{P}$ e $\mathrm{K}$.

Já Aguero et al. (2016), também testando doses de fertilizante orgânico (Bokashi) sobre mudas de bananeira da variedade "Pink Horn", tiveram resultados satisfatórios. O trabalho demonstra que, na proporção 50:50 (v/v) do substrato e do solo: bokashi, com adição de 1,5 g de Fosfato diamônico (DAP) por saco, é observado crescimento adequado da planta em relação à altura,
(B)

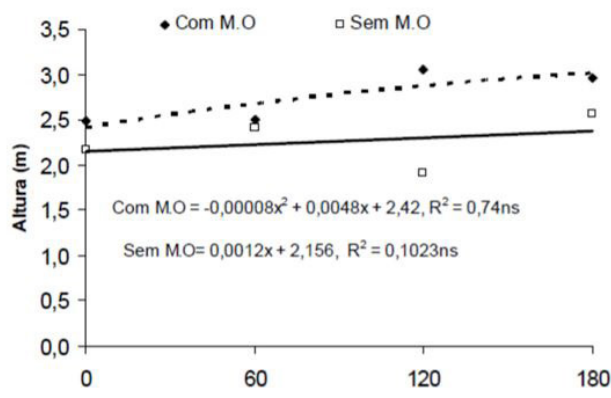

(D)

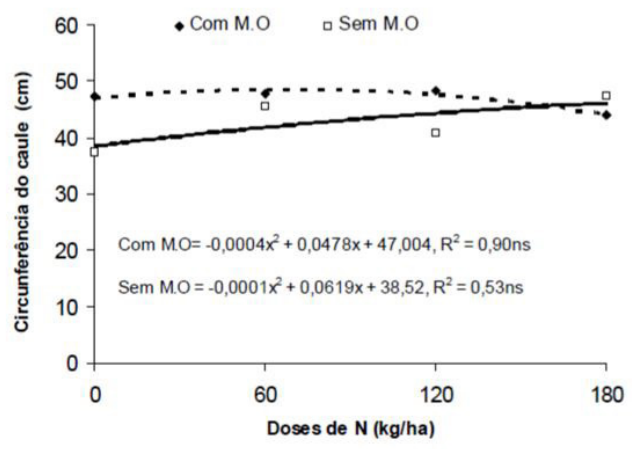

Figura 1. Altura (A e B) e diâmetro do pseudocaule (C e D) da bananeira 'nanica' em função de doses de nitrogênio aos 90 e 270 dias após o transplantio com ou sem adição de esterco bovino na cova. 


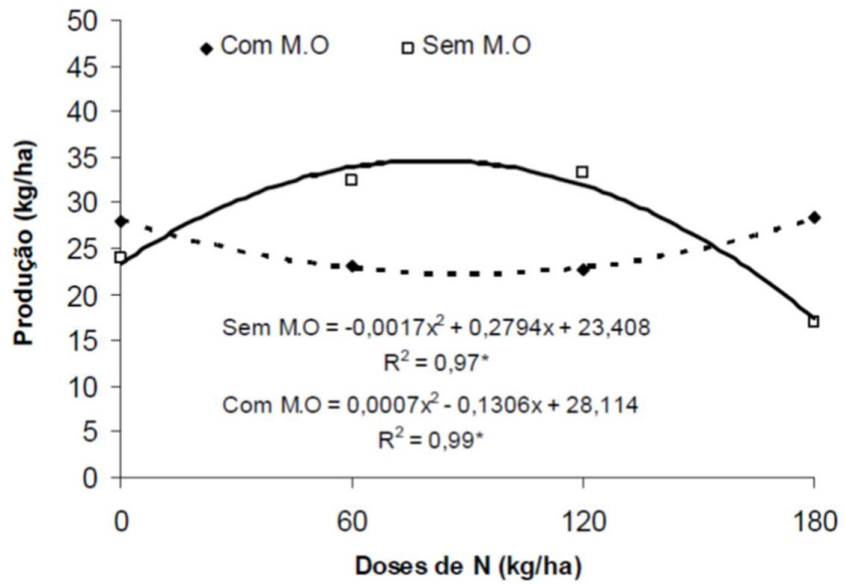

Figura 2. Produtividade da bananeira 'nanica' em função de doses de nitrogênio com ou sem adição de esterco bovino na cova.

*Valor de R significativo a 5\% pelo teste estatístico de Tukey.

diâmetro do pseudocaule e número de folhas. Além disso, as plantas obtiveram uma concentração de nutrientes semelhantes às que cresceram com o tratamento sem a aplicação do bokashi.

No tratamento sem adubação com esterco bovino, a máxima produtividade (34,9 t/ha) ocorreu com a aplicação de $82,2 \mathrm{~kg} \mathrm{~N} /$ ha. Este valor encontra-se acima do esperado para esta variedade (SILVA; FLORES; LIMA NETO, 2002), principalmente para as condições de baixa tecnologia dos pequenos produtores no semiárido. Por outro lado, a aplicação de esterco bovino, juntamente com as doses de $\mathrm{N}$, provocou decréscimos na produtividade da bananeira nas doses intermediárias de $\mathrm{N}$, cujo maior declínio ocorreu na dose de 93,3 kg N/ha. Como pode ser observado pela Figura 2, as curvas apresentadas mostram comportamento contrário entre si, indicando que o esterco bovino favoreceu o crescimento da bananeira em altura (Figura 1), mas tendeu a reduzir sua produtividade. Carvalho et al. (2020), trabalhando com bananeira BRS Platina e Prata-Anã, observaram resultados similares a este estudo, onde a produtividade dos frutos da banana adubadas com esterco bovino e farinha de osso não tiveram variações significativas em produtividade devido ao excesso de nutrientes.

Ressalta-se, contudo, que, na ausência da adubação nitrogenada, a aplicação de esterco bovino proporcionou uma produtividade correspondente a $80 \%$ da máxima produtividade obtida (34,9 kg/ha), contra $67 \%$ obtida no tratamento sem esterco bovino e sem nitrogênio. Portanto, em áreas de produção de banana 'nanica', quando a adubação com nitrogênio mineral não for realizada, a aplicação de esterco bovino, comparáveis com a dose empregada neste trabalho, poderá suprir a maior parte da necessidade da cultura em $\mathrm{N}$.

Damatto Júnior et al. (2011) observaram, trabalhando com a bananeira Prata-Anã não adubada, maior comprimento do fruto médio no primeiro ciclo, valor intermediário no segundo e menor valor no terceiro; no entanto, no quarto ciclo, com a adoção da adubação com composto orgânico, o valor de comprimento do fruto médio superou os valores obtidos nos primeiro, segundo e terceiro ciclos, em 5,2; 9,1 e $14,3 \%$, respectivamente. Santos et al. (2014), avaliando o efeito de 4 diferentes tipos de biofertilizantes sobre bananeira da variedade "Nanicão" em 3 ciclos sucessivos, observaram resultados distintos em relação a crescimento de fruto. Os autores verificaram que o crescimento médio dos frutos teve aumento no segundo ciclo em relação ao primeiro e terceiro ciclos, em 13,1\% e 12,4\%, respectivamente. Já o diâmetro dos frutos foi superior aos das plantas do primeiro e terceiro ciclos em $7,5 \%$ e $11,3 \%$, respectivamente.

O uso de substratos orgânicos consorciados com adubação mineral no cultivo da bananeira também teve resultados satisfatórios em vários estudos (CRUZ et al., 2008; STEINER; TEIXEIRA; ZECH, 2009; HUSSEIN et al., 2019). Cruz et al. (2008), testando biofertilizantes à base de esterco avícola e de resíduos de bananeira sobre o crescimento da banana ( $M$. paradisiaca AAA), verificaram que esses biofertilizantes proporcionaram aumento de nutrientes na planta, além de melhoras nas qualidades físicas e químicas do solo. $\mathrm{O}$ biofertilizante à base de esterco avícola melhorou a agregação do solo, atividade enzimática e aumento da fração de carbono lábel; já o preparado com resíduos de bananeira provocou aumento da densidade de bactérias solubilizadoras de $\mathrm{P}$, concentrações de $\mathrm{P}$ disponível no solo e maiores teores de P nas folhas.

Já Steiner, Teixeira e Zech (2009), avaliaram quatro tratamentos, compostos por adubação convencional, adubação convencional mais pó de carvão, adubação convencional mais pedações de carvão e adubação convencional mais pedaço e pó de carvão. Os autores concluíram que, em bananeira cv "Caipira", todos os tratamentos à base de carvão proporcionaram aumento parcial dos níveis foliares de $\mathrm{Ca}, \mathrm{Mg}$ e $\mathrm{S}$ de forma significativa. Os tratamentos à base de carvão também reduziram significativamente a acidez do solo em relação ao tratamento sem adição de carvão.

\section{Conclusões}

O crescimento da bananeira 'nanica' não foi afetado pela adubação nitrogenada, mas foi favorecido pela aplicação de esterco bovino. A máxima produtividade da banana 'nanica' foi obtida com a aplicação de $82,3 \mathrm{~kg} \mathrm{~N} / \mathrm{ha}$ sem a aplicação de esterco bovino.

\section{Referências}

AGUERO, R. et al. Respuesta del cultivo del plátano a diferentes proporciones de suelo y Bocashi, complementadas con fertilizante mineral en etapa de vivero. Cultivos Tropicales, La Habana, v. 37, n. 2, p. 165-174, 2016.

BORGES, A. L.; OLIVEIRA, A. M. G. Nutrição, adubação e calagem. In: CORDEIRO, Z. J. M. Banana: produção e aspectos técnicos. Brasília: EMBRAPA, 2000. p. 47-59. (Comunicação para Transferência de Tecnologia).

BORGES, A. L. et al. Adubação nitrogenada para bananeira terra (Musa sp. AAB, subgrupo Terra). Revista Brasileira de Fruticultura, Jaboticabal, v. 24, p. 189-193, 2002.

BRAZIL, E. C. et al. Desenvolvimento e produção de frutos de bananeira em resposta à adubação nitrogenada e potássica. Pesquisa Agropecuária Brasileira, Brasília, v. 35, n. 12, p. 1-14, 2000. 
CARVALHO, J. S. et al. (2020). Características fitotécnicas e nutricionais de bananeiras submetidas a fontes de fertilizantes para o manejo orgânico. Nativa, Cuiabá, v. 8, n. 3, p. 367-375, 2020.

CAVALCANTE, S. N. et al. Crescimento da planta neta de bananeira Nanicão em altura e diâmetro em função de tipos e dosagens de biofertilizantes. In: CONGRESSO BRASILEIRO DE FRUTICULTURA, 21., 2010, Natal. Anais... Natal: EMPARN/UFERSA/EMBRAPA, 2010. CD-ROM.

CRUZ, D. C. R. M. et al. Poultry manure and banana waste are effective biofertilizer carriers for promoting plant growth and soil sustainability in banana crops. Soil Biology \& Biochemistry, Missouri, v. 40, n. 12, p. 3092-3095, 2008.

DAMATTO JUNIOR, E. R. et al. Crescimento e produção da bananeira Prata-Anã adubada com composto orgânico durante cinco safras. Revista Brasileira de Fruticultura, São Paulo, p. 713-721, 2011. Volume especial.

EMPRESA BRASILEIRA DE PESQUISA AGROPECUÁRIA - EMBRAPA. Centro Nacional de Pesquisa do Solo. Sistema brasileiro de classificação de solos. 2. ed. Rio de Janeiro: Embrapa Solos, 2006. 306 p.

FOOD AND AGRICULTURE ORGANIZATION-FAO. FAOSTAT. Roma: FAO, 2017. Disponível em: <http://faostat.fao.org/site/567/ defaul.aspx\#ancor>. Acesso em: 12 jun. 2019.

GUERRA, J. G. M. et al. Uso de plantas de cobertura na valorização de processos ecológicos em sistemas orgânicos de produção na região serrana fluminense. Revista Agricultura, Rio de Janeiro, v. 4, p. 1-28, 2007.

GUIMARÃES, G. G. F. et al. Banana crop nutrition: insights into different nutrient sources and soil fertilizer application strategies. Revista Brasileira de Ciência do Solo, Viçosa, v. 44, n. e0190104, 2020.

GONÇALVES, V. D. et al. Avaliação dos cultivares de bananeira Prata-anã, Thap Maeo e Caipira em diferentes sistemas de plantio no norte de Minas Gerais. Revista Brasileira de Fruticultura, Jaboticabal, v. 30, p. 371-376, 2008.
HUSSEIN, H. S. et al. Preparation of nano-fertilizer blend from banana peels. Bulletin of the National Research Center, USA, v. 43, n. 1, p. 1-9, 2019.

INSTITUTO BRASILEIRO DE GEOGRAFIA E ESTATÍSTICA IBGE. Produção Agrícola Municipal. Rio de Janeiro: IBGE, 2019. Disponível em: <https://www.ibge.gov.br/>. Acesso em: 17 jun. 2019.

OLIVEIRA, J. M. et al. Crescimento de bananeira "Grande Naine" sob diferentes lâminas de irrigação. In: CONGRESSO BRASILEIRO DE FRUTICULTURA, 21., 2010, Natal. Anais... Jaboticabal: Sociedade Brasileira de Fruticultura, 2010. 4 p. Disponível em: $<$ http://www.alice.cnptia.embrapa.br/alice/handle/doc/873521>. Acesso em: 1 out. 2020.

OLIVEIRA, A. P.; SOUZA, C. M. Influência da cobertura morta na umidade, incidência de plantas daninhas e de broca-do-rizoma (Cosmopolites sordidus) em um pomar de bananeiras (Musa spp.). Revista Brasileira de Fruticultura, Jaboticabal, v. 25, p. 345-347, 2003.

SANTOS, J. G. R. et al. Qualidade da produção da bananeira Nanicão em função do uso de biofertilizantes. Revista Brasileira de Engenharia Agrícola e Ambiental, Campina Grande, v. 18, n. 4, p. 387-393, 2014.

SARUDI, C. et al. The of organic agriculture in rural development. Agriculture Conspectus Scientificus Polijopriredna Znanstvena, Croatia, v. 68, p. 197-202, 2003.

SILVA, J. T. A. et al. Adubação com potássio e nitrogênio em três ciclos de produção da bananeira cv. prata-anã. Revista Brasileira de Fruticultura, São Paulo, v. 25, n. 1, p. 152-155, 2003.

SILVA, S. O.; FLORES, J. C. O.; LIMA NETO, F. P. Avaliação de cultivares e híbridos de bananeira em quatro ciclos de produção. Pesquisa Agropecuária Brasileira, Brasília, v. 37, p. 1567-1574, 2002.

STATISTICAL ANALYSIS SYSTEM - SAS. System for Microsoft Windows: release 8.2. Cary: SAS, 2001. 1 CD-ROM. 